\title{
A Three Factor Remote User Authentication Scheme Using Collision Re- sist Fuzzy Extractor in Single Server Environment
}

\author{
Debasis Giri ${ }^{1}$ and Tanmoy Maitra ${ }^{2, \star}$ \\ ${ }^{1}$ Department of Computer Science and Engineering, Haldia Institute of Technology, Haldia-721657, India \\ ${ }^{2}$ Department of Computer Science and Engineering, Jadavpur University, Kolkata-700032, India
}

\begin{abstract}
Due to rapid growth of online applications, it is needed to provide such a facility by which communicators can get the services by applying the applications in a secure way. As communications are done through an insecure channel like Internet, any adversary can trap and modify the communication messages. Only authentication procedure can overcome the aforementioned problem. Many researchers have proposed so many authentication schemes in this literature. But, this paper has shown that many of them are not usable in real world application scenarios because, the existing schemes cannot resist all the possible attacks. Therefore, this paper has proposed a three factor authentication scheme using hash function and fuzzy extractor. This paper has further analyzed the security of the proposed scheme using random oracle model. The analysis shows that the proposed scheme can resist all the possible attacks. Furthermore, comparison between proposed scheme and related existing schemes shows that the proposed scheme has better trade-off among storage, computational and communication costs.
\end{abstract}

\section{Introduction}

Nowadays, online applications like bill payment, banking system, telecare medical system, social networking, e-voting and so on are rapidly used for their easy and efficient access. All the applications are going through a client/server environment and communications are done through public channel like Internet because of availability of public bandwidth. Therefore, all communication messages of the applications are public. As a result, any one can trap and modify the communication messages. For this purpose, in such communication system, authentication scheme is rapidly used by which after verifying the communicators and their messages, a secure communication can be done through public channel. In this regard, smart card and password based user authentication scheme is very much popular for online communication system. However, a suitable smart card based authentication scheme should satisfy the following property:

Low cost: Computational cost, communication cost and smart card storage cost are three basic network parameters to measure performances of an authentication scheme. Therefore, it has to considered that these three parameters are reduced as much possible when an authentication scheme is going to be designed.

Prevention of security attacks: During communication through public channel, it is needed to secure the message from outsider adversary. An authentication scheme needs to be designed such a way by which from the communication messages, any adversary will unable to extract useful information and the scheme can resist security attacks.

Session key agreement: After authentication in both ways, a common secret session key is needed to carry on the communications within the same session after encrypting the paintext messages by the session key.

Mutual authentication: Mutual authentication is a essential property for an authentication scheme by which all the communicators can authenticate or verify each other.

Efficient login procedure: For an authentication scheme, it is needed that smart card checks the wrong inputs before going to send a login message to server. By checking wrong inputs in login phase, extra communication overhead can be avoided. Therefore, it is crucial property of a good authentication scheme.

^e-mail: tanmoy.maitra@live.com 
Efficient password change procedure: A valid user can change the password freely and securely without taking any help from server. For this purpose, the smart card should verify the old password in the password change phase, so that an unauthorized user cannot change the authorized user's password even if it gets the valid users' smart card. The afore mentioned property should present in an authentication scheme.

Traceability: In an authentication scheme, it is also needed to trace the sender of a message for corresponding receiver [1]. Otherwise, any one can mount denial of service (DoS) attack.

Furthermore, to enhanced the security of password based authentication scheme, biometric feature (i.e, finger print, ires, retina etc.) [2] is added with the password. Therefore, this research focus to design a biometric plus password based efficient authentication scheme by considering all the afore mentioned properties.

\subsection{Literature Survey}

A brief survey of existing authentication schemes is described in this section. First Lamport [3] proposed a password-based authentication scheme based on one way hash function. However, Shimizu et al. [4] showed that the Lamport's scheme [3] suffers from different attacks. After that so many remote user authentication schemes [5-16] have been proposed in this regard which are based on only password. But, the researchers have considered biometric feature [2] with the password to enhance the security label. Therefore, many researchers have proposed biometric and password based authentication schemes in [17-28]. Li and Hwang [17] proposed a biometrics-based remote user authentication scheme in 2010. However, in 2011, Das [18] showed that Li and Hwang's scheme [17] had flaws in the login phase, authentication phase and password change phase and therefore, Das also proposed an authentication scheme. An [19] showed that Das's scheme [18] cannot resist the server masquerading attack, user impersonation attack, password guessing attack and insider attack, and so proposed an improved scheme. Li et al. [20] found that An's scheme [19] suffered from the denial-of-service (DoS) attack, the forgery attack and also did not provide forward secrecy. Furthermore, in 2013, Lee and Hsu [21] pointed out that Das's scheme [18] is also suffering from privileged insider attack and the off-line password guessing attack. Therefore, Lee and Hsu [21] proposed a biometric based authentication scheme to overcome the weaknesses of Das's scheme [18]. In 2013, Tan [22] proposed a three-factor authentication scheme. But, Yan et al. [23] pointed out that Tan's scheme [22] is vulnerable to the Denial-of-Service (DoS) attack. However, recently, Mishra et al. [24] showed that Yan et al.'s scheme [23] suffers from off-line password guessing attack and has inefficient login and password change phases. Huang et al. [29] proposed an authentication scheme based on RSA. But Amin et al. [30] proved that Huang et al.'s scheme [29] unable to protect forgery attack and also introduced an authentication protocol in [30].

\subsection{Contribution}

This paper proposes a three factor authentication scheme using hash function and fuzzy extractor, where three factor means (1) users' password, (2) users' biometric and (3) smart card. This paper further analyzes the security of the proposed scheme using random oracle model. The analysis shows that an adversary cannot mount any attacks on the proposed scheme due to hardness of inversion of one-way hash function as well as it has to solve hardness of fuzzy factor. Furthermore, comparison between proposed scheme and related existing schemes shows that the proposed scheme has better trade-off among storage, computational and communication costs. It is a great contribution that the proposed scheme resists all the possible attacks with better trade-off among different costs.

\subsection{Road Map}

This section describes a road map which has been followed throughout this paper. Section 2 briefly introduces some preliminary mathematical concepts for introducing the proposed scheme. Section 3 describes a network model and an adversary model to analyze the proposed scheme. A proposed scheme is described in section 4 . Section 5 describes cryptanalysis of the proposed scheme and Section 6 compares the performances of the proposed scheme with previously published schemes. Conclusion of this paper appears in section 7.

\section{Preliminaries}

In this section, a briefly review the basic concepts of cryptographic one-way hash function and collision resist fuzzy extractor are introduced.

Definition 1. A collision resistant cryptographic one-way hash function [25, 27] maps a string of arbitrary length to a string of fixed length called the hashed value. It can be symbolized as: $H: A \rightarrow B$, where $A$ is a binary string of 
arbitrary length and $B$ is a binary string of fixed length $n$. If $A d v_{\mathcal{A}}^{H}\left(t_{1}\right)$ is the advantage to an adversary $\mathcal{A}$ to choose a random pair $(a, b) \in A \times A$ such that $H(a)=H(b)$, where $a \neq b$ for the time duration $t_{1}$, it can be considered that $A d v_{\mathcal{A}}^{H}\left(t_{1}\right)$ is the probability in the advantage which is computed over the random choices made by the adversary $\mathcal{A}$ for the time duration $t_{1}$. Then the cryptographic one-way hash function $H(\cdot)$ is called collision-resistant, if $A d v_{\mathcal{A}}^{H}\left(t_{1}\right) \leq \xi_{1}$, for any small $\xi_{1}>0$. $A d v_{\mathcal{A}}^{H}\left(t_{1}\right)$ is represented as:

$$
A d v_{\mathcal{A}}^{H}\left(t_{1}\right)=\operatorname{Pr}\left[(a, b) \in_{R} A \times A \mid(a \neq b) \wedge H(a)=H(b)\right],
$$

where $\operatorname{Pr}[\mathcal{E}]$ denotes the random event $\mathcal{E}$.

Definition 2. A collision resistant fuzzy extractor $[2,27]$ can be model as a procedure, known as Gen, which takes a binary string say, $B$ of some metric space $M$ as an input, where $M \in\{0,1\}^{k}$, for some $k$ bits and produces a random string say, $\phi \in_{R}\{0,1\}^{n}$, for some $n$ bits and an auxiliary string say, $\theta \in_{R}\{0,1\}^{r}$, for some $r$ bits, where $r=k$ or $n$ bits. It can be mathematically represented by Gen : $M \rightarrow \phi \times \theta$. Another procedure, known as Rep, takes a binary string say, $B^{\prime}$ of the metric space $M \in\{0,1\}^{k}$, where $B \neq B^{\prime}$ and a uniform distribution binary string

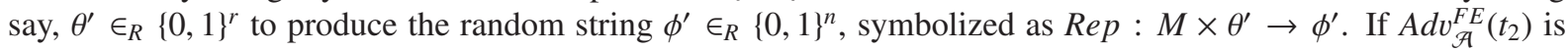
the advantage to an adversary $\mathcal{A}$ to choose a pair $\left(B, B^{\prime}\right) \in_{R} M \times M$ randomly such that $\operatorname{des}\left(B, B^{\prime}\right) \leq \delta d$, Gen $(B)$ $=\operatorname{Gen}\left(B^{\prime}\right)$ and $\operatorname{Rep}(B, \theta)=\operatorname{Rep}\left(B^{\prime}, \theta^{\prime}\right)$, where $\delta d$ is the difference tolerance level and $B \neq B^{\prime}$ for the time duration $t_{2}$, it can be considered that $A d v_{\mathcal{A}}^{F E}\left(t_{2}\right)$ is the probability that the advantage is computed over the random choices made by $\mathcal{A}$ for the time duration $t_{2}$. Then the fuzzy extractor $F E$ is called collision-resistant, if $A d v_{\mathcal{A}}^{F E}\left(t_{2}\right) \leq \xi_{2}$, for any small $\xi_{2}>0 . A d v_{\mathcal{A}}^{F E}\left(t_{2}\right)$ is represented as:

$$
\begin{array}{r}
A d v_{\mathcal{A}}^{E F}\left(t_{2}\right)=\operatorname{Pr}\left[\left(B, B^{\prime}\right) \in_{R} M \times M \mid\left(B \neq B^{\prime}\right) \wedge \operatorname{des}\left(B, B^{\prime}\right) \leq \delta d\right. \\
\left.\wedge \operatorname{Gen}(B)=\operatorname{Gen}\left(B^{\prime}\right) \wedge \operatorname{Rep}(B, \theta)=\operatorname{Rep}\left(B^{\prime}, \theta^{\prime}\right)\right],
\end{array}
$$

for all probabilistic polynomial-time algorithms Gen and Rep.

\section{Model}

This section will introduce two following models:

Network Model: Architecture of the proposed scheme is shown in Figure 1 where, users have to register to a remote server to get their smart card which is known as registration procedure (see Figure 1(a)). Whenever the registered users want to get service from the remote server by accessing their smart card through public channel like Internet, the smart card sends a login request message to the remote server. After verifying the login request message, the remote server sends corresponding reply message to the sender. After receiving the reply message, the corresponding smart card checks the validity of the reply message. Upon receiving correct reply message, both user and the remote server agree for a shared secret session key (See Figure 1(b)).

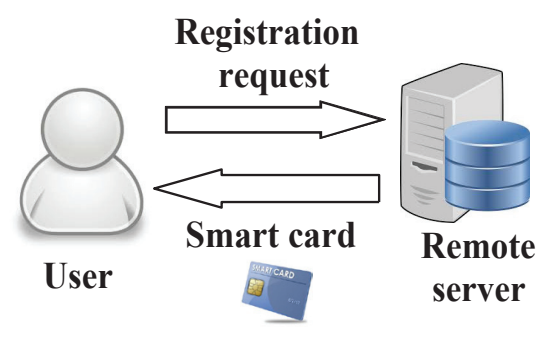

(a)

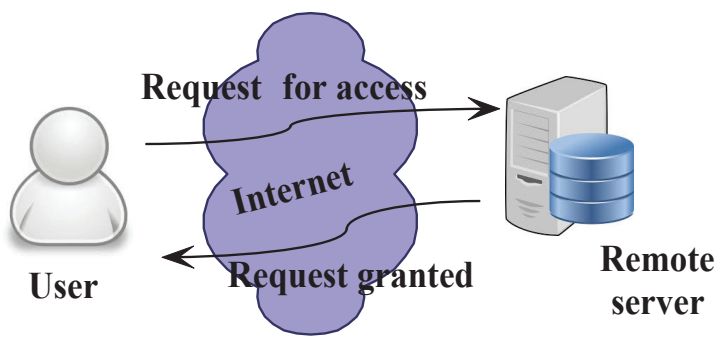

(b)

Figure 1. Network architecture of proposed scheme (a) registration procedure and (b) login and authentication procedure

Adversary Model: To analyze the security of the proposed scheme, Dolev-Yao threat model [31] has been considered in which the communicating parties communicate through an insecure channel. Therefore, an adversary $\mathcal{A}$ can trap the transmitted messages over the public or insecure channel, and furthermore he/she can modify, delete or change the contents of the transmitted messages. The adversary $\mathcal{A}$ also obtains the information which are stored 
in the user's smart card by monitoring the power consumption $[32,33]$. Generally, identity and password of the user are low entropy in cryptography, that means the adversary can guess the identity and password individually using dictionary attack in polynomial time. But, the adversary cannot guess identity and password simultaneously in on-line/off-line within a polynomial time as pointed out in [34]. According to our adversary model, we consider two following cases:

- Case 1. A third party from outside of the system tries to mount various attacks on authentication system as an adversary $\mathcal{A}$.

- Case 2. A registered user from inside of the system tries to extract secret information of the server by which he/she can mount various attacks on authentication system as an other user or adversary $\widehat{\mathcal{A}}$.

\section{Proposed Scheme}

This section proposes an authentication scheme. A nomenclature is given in Table 1 to introduce the proposed scheme. The proposed scheme consists of five phases namely, 1) initialization phase, 2) registration phase, 3) login phase, 4) authentication and key agreement phase and 5) password update phase. The phases are as follows:

Table 1. Nomenclature

\begin{tabular}{ll}
\hline Term & Usage \\
\hline$U_{i}$ & i-th Patient \\
$R S$ & Remote server \\
$p w_{i}$ & Password of user $U_{i}$ \\
$I D_{i}$ & Identity of user $U_{i}$ \\
$B_{i}$ & Biometric parameter of $U_{i}$ \\
$r_{i}$ & Random number chosen by smart card \\
$y_{i}$ & Random number chosen by $R S$ \\
$T$ & Current timestamp \\
$d e s(\cdot)$ & Distance measurement function \\
$\delta d$ & Estimated difference \\
$X^{\prime}$ & Parameter $X$ computed or extracted by smart card \\
$X^{*}$ & Parameter $X$ computed or extracted by $R S$ \\
$\delta T$ & Estimated timestamp \\
$S K_{i}$ & Shared session key between $U_{i}$ and $R S$ \\
$H(\cdot)$ & Cryptographic one-way hash function \\
$S$ & Secret key of remote server \\
$\|$ & Concatenation operation \\
$\oplus$ & Bit wise XOR operation
\end{tabular}

\subsection{Initialization Phase}

A remote server $R S$ runs algorithm $\mathscr{G}$ to compute a large prime number $q$. Then it selects a random number $s$ such that $s \in_{R} Z_{q}^{*}$. It further chooses a collision resist cryptographic one-way hash function $H:\{0,1\}^{*} \rightarrow\{0,1\}^{n}$, where $n$ is a fixed length integer number. Finally, $R S$ publishes $H(\cdot)$ as public and keeps $s$ as secret.

\subsection{Registration Phase}

Whenever a new user $U_{i}$ wants to register to the remote server $R S$, this phase is invoked. This phase is as follows:

1. The user inputs their biometric feature (i.e., finger print) to a sensor. The sensor generates a corresponding biometric information $B_{i}$ and provides it to $U_{i}$.

2. The user $U_{i}$ chooses an identity $I D_{i}$, password $p w_{i}$ and generates an unique pair $\left(\theta_{i}, \phi_{i}\right)$ from $B_{i}$ by computing $\left(\phi_{i}, \theta_{i}\right) \leftarrow \operatorname{Gen}\left(B_{i}\right) . U_{i}$ then computes $p w r_{i}=H\left(p w_{i} \| \phi_{i}\right)$ and sends $\left\langle I D_{i}, p w r_{i}\right\rangle$ to $R S$ through a secure channel. 


\begin{tabular}{|c|c|}
\hline$U_{i}$ & $R S$ \\
\hline \multicolumn{2}{|l|}{$\begin{array}{l}\text { Input biometrics to a sensor to get } \\
\text { biometric information } B_{i} \\
\text { Choose } I D_{i} \text { and } p w_{i} \\
\text { Compute }\left(\phi_{i}, \theta_{i}\right) \leftarrow G e n\left(B_{i}\right) \\
\text { Calculate } p w r_{i}=H\left(p w_{i} \| \phi_{i}\right)\end{array}$} \\
\hline$\left\langle I D_{i}, p w r_{i}\right\rangle$ & Compute $A_{i}=H\left(s \| I D_{i}\right), D_{i}=H\left(A_{i}\right)$ \\
\hline Calculate $\widehat{B}_{i}=B_{i} \oplus H\left(I D_{i} \| p w_{i}\right)$ & $\begin{array}{l}\text { Check } D_{i}=\text { ? database } \\
\text { Calculate } C_{i}=A_{i} \oplus p w r_{i} \text {, store } D_{i} \text { into its database } \\
\left\langle C_{i}, D_{i}, \operatorname{des}(\cdot)\right\rangle\end{array}$ \\
\hline$\widehat{\theta_{i}}=\theta_{i} \oplus H\left(I D_{i} \| p w_{i}\right)$ & \\
\hline Reserve $\left\langle C_{i}, D_{i}, \widehat{B_{i}}, \widehat{\theta}_{i}, \operatorname{des}(\cdot)\right\rangle$ into smart card & $\rightarrow$ Secure channel \\
\hline
\end{tabular}

Figure 2. Registration phase of the proposed scheme

3. After getting a registration request $\left\langle I D_{i}, p w r_{i}\right\rangle$ from $U_{i}, R S$ computes $A_{i}=H\left(s \| I D_{i}\right)$ and $D_{i}=H\left(A_{i}\right)$. RS then checks $D_{i}$ is present in its database or not. If it is present in its database, $R S$ sends a decline message to $U_{i}$ because, $I D_{i}$ is already used by another user. Therefore, the user $U_{i}$ has to choose another identity until fresh identity is not obtained. If $D_{i}$ is not present in its database, $R S$ computes $C_{i}=A_{i} \oplus p w r_{i}$ and stores parameters $\left\langle C_{i}, D_{i}\right.$, des $\left.(\cdot)\right\rangle$ into the memory of smart card, where $\operatorname{des}(\cdot)$ is a distance measurement function. Then $R S$ issues the smart card for $U_{i}$ and sends it through a secure channel or by person. $R S$ then updates its database by adding $D_{i}$ into the list.

4. After getting the smart card, $U_{i}$ inserts it into a terminal or card reader and submits their identity $I D_{i}$ and password $p w_{i}$.

5. The smart card computes $\widehat{B_{i}}=B_{i} \oplus H\left(I D_{i} \| p w_{i}\right), \widehat{\theta_{i}}=\theta_{i} \oplus H\left(I D_{i} \| p w_{i}\right)$. Finally, the smart card stores $\left\langle\widehat{B_{i}}, \widehat{\theta_{i}}\right\rangle$ into its memory. Note that, smart card stored parameters are $\left\langle C_{i}, D_{i}, \widehat{B}_{i}, \widehat{\theta}_{i}, \operatorname{des}(\cdot)\right\rangle$.

Figure 2 shows the registration phase of the proposed scheme.

\subsection{Login Phase}

Whenever a registered user $U_{i}$ wants to access the remote server, this phase is invoked. $U_{i}$ inserts their smart card into a card reader or terminal and provides their biometric information $B_{i}^{*}$ through sensor, identity $I D_{i}$ and password $p w_{i}$ to the smart card. The smart card then executes following steps:

1. The smart card computes $B_{i}^{\prime}=\widehat{B}_{i} \oplus H\left(I D_{i} \| p w_{i}\right)$ and checks $\operatorname{des}\left(B_{i}^{*}, B_{i}^{\prime}\right) \leq \delta d$. If it does not hold, the smart card rejects $U_{i}$; otherwise, it follows next step.

2. The smart card computes $\theta_{i}^{\prime}=\widehat{\theta_{i}} \oplus H\left(I D_{i} \| p w_{i}\right), \phi_{i}^{\prime} \leftarrow \operatorname{Rep}\left(B_{i}^{*}, \theta_{i}^{\prime}\right), p w r_{i}^{\prime}=H\left(p w_{i} \| \phi_{i}^{\prime}\right), A_{i}^{\prime}=C_{i} \oplus p w r_{i}^{\prime}, D_{i}^{\prime}$ $=H\left(A_{i}^{\prime}\right)$ and checks computed $D_{i}^{\prime}$ and stored $D_{i}$ are equal or not. If equality does not hold, the smart card rejects $U_{i}$; otherwise, it follows next step.

3. The smart card chooses a random number $r_{i} \in_{R} Z_{q}^{*}$ and further computes $E_{i}=r_{i} \oplus A_{i}^{\prime}, G_{i}=T_{i}^{1} \oplus A_{i}^{\prime} \oplus H\left(r_{i}\right)$ and $F_{i}=H\left(r_{i}\left\|A_{i}^{\prime}\right\| T_{i}^{1}\right)$, where $T_{i}^{1}$ is the current login timestamp of $U_{i}$. The user $U_{i}$ then sends a login request message $\left\langle I D_{i}, G_{i}, F_{i}, E_{i}\right\rangle$ to the registration server $R S$ through a public channel.

Figure 3 shows the login phase of the proposed scheme.

\subsection{Authentication and Key Agreement Phase}

After receiving the login request message $\left\langle I D_{i}, G_{i}, F_{i}, E_{i}\right\rangle$ from the user $U_{i}$ at timestamp $T_{s}$, the remote server $R S$ computes following steps:

1. $R S$ computes $A_{i}^{*}=H\left(s \| I D_{i}\right), r_{i}^{*}=A_{i}^{*} \oplus E_{i}, T_{i}^{1^{*}}=A_{i}^{*} \oplus G_{i} \oplus H\left(r_{i}^{*}\right)$ and checks $\left(T_{s}-T_{i}^{1^{*}}\right) \leq \delta T$. It it does not hold, $R S$ rejects $U_{i}$; otherwise, executes the next step. 


\begin{tabular}{|c|c|c|}
\hline $\begin{array}{c}U_{i} \\
\text { Input } I D_{i}, p w_{i} \text { and biometric } B_{i}^{*}\end{array}$ & Terminal & $R S$ \\
\hline \multirow{10}{*}{ Input $I D_{i}, p w_{i}$ and biometric $B_{i}^{*}$} & & \\
\hline & Compute $B_{i}^{\prime}=\widehat{B}_{i} \oplus H\left(I D_{i} \| p w_{i}\right)$ & \\
\hline & Check $\operatorname{des}\left(B_{i}^{*}, B_{I}^{\prime}\right) \leq \delta d$ & \\
\hline & Compute $\theta_{i}^{\prime}=\widehat{\theta}_{i} \oplus H\left(I D_{i} \| p w_{i}\right), \phi_{i}^{\prime} \leftarrow \operatorname{Rep}\left(B_{i}^{*}, \theta_{i}^{\prime}\right)$ & \\
\hline & $p w r_{i}^{\prime}=H\left(p w_{i} \| \phi_{i}^{\prime}\right), A_{i}^{\prime}=C_{i} \oplus p w r_{i}^{\prime}$ & \\
\hline & $D_{i}^{\prime}=H\left(A_{i}^{\prime}\right)$ & \\
\hline & Check computed $D_{i}^{\prime}=$ ? stored $D_{i}$ & \\
\hline & Compute $F-r . u^{\prime} C_{-}-T^{1} u^{\prime} H_{(r)}$ & \\
\hline & $F_{i}=H\left(r_{i}\left\|A^{\prime}\right\| T^{1}\right)$ & \\
\hline & $\left\langle I D_{i}, G_{i}, F_{i}, E_{i}\right\rangle$ & $\rightarrow$ Insecure channel \\
\hline
\end{tabular}

Figure 3. Login phase of the proposed scheme

\begin{tabular}{|ll|}
\hline \multicolumn{1}{|c|}{ Terminal } & \multicolumn{1}{c|}{$R S$} \\
\hline$\left\langle I D_{i}, G_{i}, F_{i}, E_{i}\right\rangle$ & Compute $A_{i}^{*}=H\left(s \| I D_{i}\right), T_{i}^{1^{*}}=A_{i}^{*} \oplus G_{i}$ \\
& Check $\left(T_{s}-T_{i}^{1^{*}}\right) \leq \delta T$ \\
& Compute $r_{i}^{*}=A_{i}^{*} \oplus E_{i}, F_{i}^{*}=H\left(r_{i}^{*}\left\|A_{i}^{*}\right\| T_{i}^{1^{*}}\right)$ \\
& Check computed $F_{i}^{*}=$ ? received $F_{i}$ \\
& Chooses a random number $y_{i} \in_{R} Z_{q}^{*}$ \\
Compute $y_{i}^{\prime}=A_{i}^{\prime} \oplus Q_{i}, T_{s}^{\prime}=A_{i}^{\prime} \oplus K_{i} \oplus y_{i}^{\prime}$ & Compute $Q_{i}=A_{i}^{*} \oplus y_{i}, K_{i}=T_{s} \oplus A_{i}^{*} \oplus H\left(y_{i}\right)$ \\
Check $\left(T_{i}^{2}-T_{s}^{\prime}\right) \leq \delta T$ & $\left\langle L_{i}=H\left(T_{s}\left\|S K_{i}\right\| A_{i}^{*}\right)\right.$ \\
Compute $S K_{i}^{\prime}=H\left(y_{i}^{\prime} \| r_{i}\right)$ & $\left.\stackrel{ }{ }, K_{i}\right\rangle$ \\
$L_{i}^{\prime}=H\left(T_{s}^{\prime}\left\|S K_{i}^{\prime}\right\| A_{i}^{\prime}\right)$ & \\
Check computed $L_{i}^{\prime}=$ ? received $L_{i}$ & \\
$U_{i}$ agrees upon the shared secret key $S K_{i}$ & \\
\hline
\end{tabular}

Figure 4. Authentication and key agreement phase of the proposed scheme

2. $R S$ computes $F_{i}^{*}=H\left(r_{i}^{*}\left\|A_{i}^{*}\right\| T_{i}^{1^{*}}\right)$ and checks computed $F_{i}^{*}$ and received $F_{i}$ are equal or not. If it does not hold, $R S$ rejects login request message of $U_{i}$; otherwise, follows the next step.

3. $R S$ chooses a random number $y_{i} \in_{R} Z_{q}^{*}$ and further computes $Q_{i}=A_{i}^{*} \oplus y_{i}, K_{i}=T_{s} \oplus A_{i} \oplus H\left(y_{i}\right), S K_{i}=$ $H\left(y_{i} \| r_{i}^{*}\right), L_{i}=H\left(T_{s}\left\|S K_{i}\right\| A_{i}^{*}\right)$ and sends a reply message $\left\langle Q_{i}, L_{i}, K_{i}\right\rangle$ to $U_{i}$ through a public channel. $R S$ accepts $S K_{i}$ as a shared secret session key.

After receiving the reply message $\left\langle Q_{i}, L_{i}, K_{i}\right\rangle$ from $R S$ at timestamp $T_{i}^{2}$, the smart card of the user $U_{i}$ further executes the following steps to verify the reply message of $R S$ :

1. The smart card computes $y_{i}^{\prime}=A_{i}^{\prime} \oplus Q_{i}, T_{s}^{\prime}=A_{i}^{\prime} \oplus K_{i} \oplus H\left(y_{i}^{\prime}\right)$ and checks $\left(T_{i}^{2}-T_{s}^{\prime}\right) \leq \delta T$. It it does not hold, the smart card rejects the reply message; otherwise, executes the next step.

2. The smart card computes $S K_{i}^{\prime}=H\left(y_{i}^{\prime} \| r_{i}\right), L_{i}^{\prime}=H\left(T_{s}^{\prime}\left\|S K_{i}^{\prime}\right\| A_{i}^{\prime}\right)$ and checks computed $L_{i}^{\prime}$ and received $L_{i}$ are equal or not. If they are equal, the user $U_{i}$ agrees upon the shared secret key $S K_{i}$; otherwise, rejects the reply message.

Figure 4 shows the authentication and key agreement phase of the proposed scheme.

\subsection{Password Update Phase}

Whenever a user $U_{i}$ wants to change their password, this phase is invoked. $U_{i}$ inserts their smart card into a card reader or terminal and provides their biometric information $B_{i}^{*}$ through sensor, identity $I D_{i}$ and password $p w_{i}$ to the smart card. The smart card then executes following steps:

1. The smart card computes $B_{i}^{\prime}=\widehat{B}_{i} \oplus H\left(I D_{i} \| p w_{i}\right)$ and checks $\operatorname{des}\left(B_{i}^{*}, B_{I}^{\prime}\right) \leq \delta d$. If it does not hold, the smart card rejects $U_{i}$; otherwise, it follows next step. 


\begin{tabular}{|c|c|}
\hline$U_{i}$ & Terminal \\
\hline \multicolumn{2}{|l|}{ Input $I D_{i}, p w_{i}$ and biometric $B_{i}$} \\
\hline & Compute $B_{i}^{\prime}=\widehat{B}_{i} \oplus H\left(I D_{i} \| p w_{i}\right)$ \\
\hline & Check $\operatorname{des}\left(B_{i}^{*}, B_{I}^{\prime}\right) \leq \delta d$ \\
\hline & $\theta_{i}^{\prime}=\widehat{\theta_{i}} \oplus H\left(I D_{i} \| p w_{i}\right), \phi_{i}^{\prime} \leftarrow \operatorname{Rep}\left(B_{i}^{*}, \theta_{i}^{\prime}\right)$ \\
\hline & $p w r_{i}^{\prime}=H\left(p w_{i} \| \phi_{i}^{\prime}\right), A_{i}^{\prime}=C_{i} \oplus p w r_{i}^{\prime}$ \\
\hline & $D_{i}^{\prime}=H\left(A_{i}^{\prime}\right)$ \\
\hline & Check computed $D_{i}^{\prime}=$ ? stored $D_{i}$ \\
\hline \multirow[t]{6}{*}{ Input a new $p w_{i}^{[n e w]}$} & \\
\hline & Compute $p w r_{i}^{[n e w]}=H\left(p w_{i}^{[n e w]} \| \phi_{i}^{\prime}\right)$ \\
\hline & $C_{i}^{[n e w]}=A_{i}^{\prime} \oplus p w r_{i}^{[n e w]}, \widehat{B_{i}^{[n e w]}}=B_{i}^{\prime} \oplus H\left(I D_{i} \| p w_{i}^{[\text {new }]}\right)$ \\
\hline & $\overrightarrow{\theta_{i}^{[n e w]}}=\theta_{i}^{\prime} \oplus H\left(I D_{i} \| p w_{i}^{[\text {new }]}\right)$ \\
\hline & store $C_{i}^{[\text {new] }}, \widehat{B_{i}^{[n e w]}}, \widehat{\theta_{i}^{[n e w]}}$ \\
\hline & by replacing $C_{i}, \widehat{B}_{i}, \widehat{\theta}_{i}$ \\
\hline
\end{tabular}

Figure 5. Password update phase of the proposed scheme

2. The smart card computes $\theta_{i}^{\prime}=\widehat{\theta_{i}} \oplus H\left(I D_{i} \| p w_{i}\right), \phi_{i}^{\prime} \leftarrow \operatorname{Rep}\left(B_{i}^{*}, \theta_{i}^{\prime}\right), p w r_{i}^{\prime}=H\left(p w_{i} \| \phi_{i}^{\prime}\right), A_{i}^{\prime}=C_{i} \oplus p w r_{i}^{\prime}, D_{i}^{\prime}$ $=H\left(A_{i}^{\prime}\right)$ and checks computed $D_{i}^{\prime}$ and stored $D_{i}$ are equal or not. If equality does not hold, the smart card rejects $U_{i}$; otherwise, gives permission to enter their new password.

3. The user $U_{i}$ selects their new password $p w_{i}^{[n e w]}$ and proves it to the smart card. The smart card then further proceeds to next step.

4. The smart card computes $p w r_{i}^{[\text {new }]}=H\left(p w_{i}^{[\text {new }]} \| \phi_{i}^{\prime}\right), C_{i}^{[\text {new }]}=A_{i}^{\prime} \oplus p w r_{i}^{[\text {new }]}, \widehat{B_{i}^{[n e w]}}=B_{i}^{\prime} \oplus H\left(I D_{i} \| p w_{i}^{[\text {new }]}\right)$ and $\widehat{\theta_{i}^{[n e w]}}=\theta_{i}^{\prime} \oplus H\left(I D_{i} \| p w_{i}^{[\text {new }]}\right)$. The smart card then stores $C_{i}^{[\text {new }]}, \widehat{B_{i}^{[n e w]}}$ and $\widehat{\theta_{i}^{[n e w]}}$ in the place of $C_{i}, \widehat{B_{i}}$ and $\widehat{\theta_{i}}$ respectively into the memory of smart card.

Figure 5 shows the password update phase of the proposed scheme.

\section{Security Analysis of Proposed Scheme}

The formal security analysis of the proposed scheme under the random oracle model is presented in this section. This security analysis uses the formal security analysis under the generic group model of cryptography. In the following, this work defines random oracles for the formal security analysis of the proposed scheme:

- Oracle $\mathcal{H}$ is a random oracle which maintains a tuple $\langle x, y\rangle$ such that $y=H(x)$. It returns $x$ from $y$ upon receiving a query $(q H, y)$ if $\langle x, y\rangle$ is present in the tuple; otherwise returns a random number $r_{1}$. Then it stores a new entry $\left\langle r_{1}, y\right\rangle$ into its tuple.

- Oracle $\mathcal{F} \mathcal{E}$ is a random oracle which contains two parts:

1.Oracle $\mathcal{F} \mathcal{E}_{G e n}$ unconditionally outputs the pair $(\phi, \theta)$ from the corresponding tuple $\langle B, \phi, \theta\rangle$ upon receiving a query $(q \operatorname{Gen}, B)$ such that $(\phi, \theta) \leftarrow \operatorname{Gen}(B)$ if $\langle B, \phi, \theta\rangle$ is present in its tuple; otherwise returns two random numbers $r_{2}$ and $r_{3}$. Then it stores new entry $\left\langle B, r_{2}, r_{3}\right\rangle$ into its tuple.

2.Oracle $\mathcal{F} \mathcal{E}_{R e p}$ unconditionally outputs $\phi$ from the corresponding tuple $\left\langle B^{\prime}, \phi, \theta\right\rangle$ upon receiving a query ( $q$ Rep $\left., B^{\prime}, \theta\right)$ such that $\phi \leftarrow \operatorname{Rep}\left(B^{\prime}, \theta\right)$ if $\left\langle B^{\prime}, \phi, \theta\right\rangle$ is present in its tuple; otherwise returns random number $r_{4}$. Then it stores new entry $\left\langle B^{\prime}, r_{4}, \theta\right\rangle$ into its tuple.

Theorem 1. Under the assumption that a cryptographic one-way hash function $H(\cdot)$ and fuzzy extractor $F E$ act as random oracles, the proposed scheme is provably secure against an adversary $\mathcal{A}$ for deriving the password pwi and biometric parameter $B_{i}$ of a user $U_{i}$ even if the adversary $\mathcal{A}$ gets parameters that are stored into the memory of $U_{i}$ 's smart card and traps the communication messages between $U_{i}$ and the remote server $R S$.

Proof 1. This research construct an adversary $\mathcal{A}$ who has the ability to derive the password $p w_{i}$ and biometric parameter $B_{i}$ of a user $U_{i}$. For this purpose, this research assumes that the smart card of a user $U_{i}$ is lost or stolen. Thus, the adversary $\mathcal{A}$ can extract the stored parameters $\left\langle C_{i}, D_{i}, \widehat{B_{i}}, \widehat{\theta_{i}}\right\rangle$ from the memory of the smart card 
of the user $U_{i}$ by power monitoring [32][33]. The adversary $\mathcal{A}$ also traps login request message $\left\langle I D_{i}, G_{i}, F_{i}, E_{i}\right\rangle$ and a reply message $\left\langle Q_{i}, L_{i}, K_{i}\right\rangle$. The adversary $\mathcal{A}$ runs the experiment, EXP $1_{\mathcal{A} \text {, TFUAS }}^{\text {orace }}$ for our three factor user authentication scheme (TFUAS) to derive the password $p w_{i}$ and biometric parameter $B_{i}$ of the user $U_{i}$ as given in the Algorithm 1.

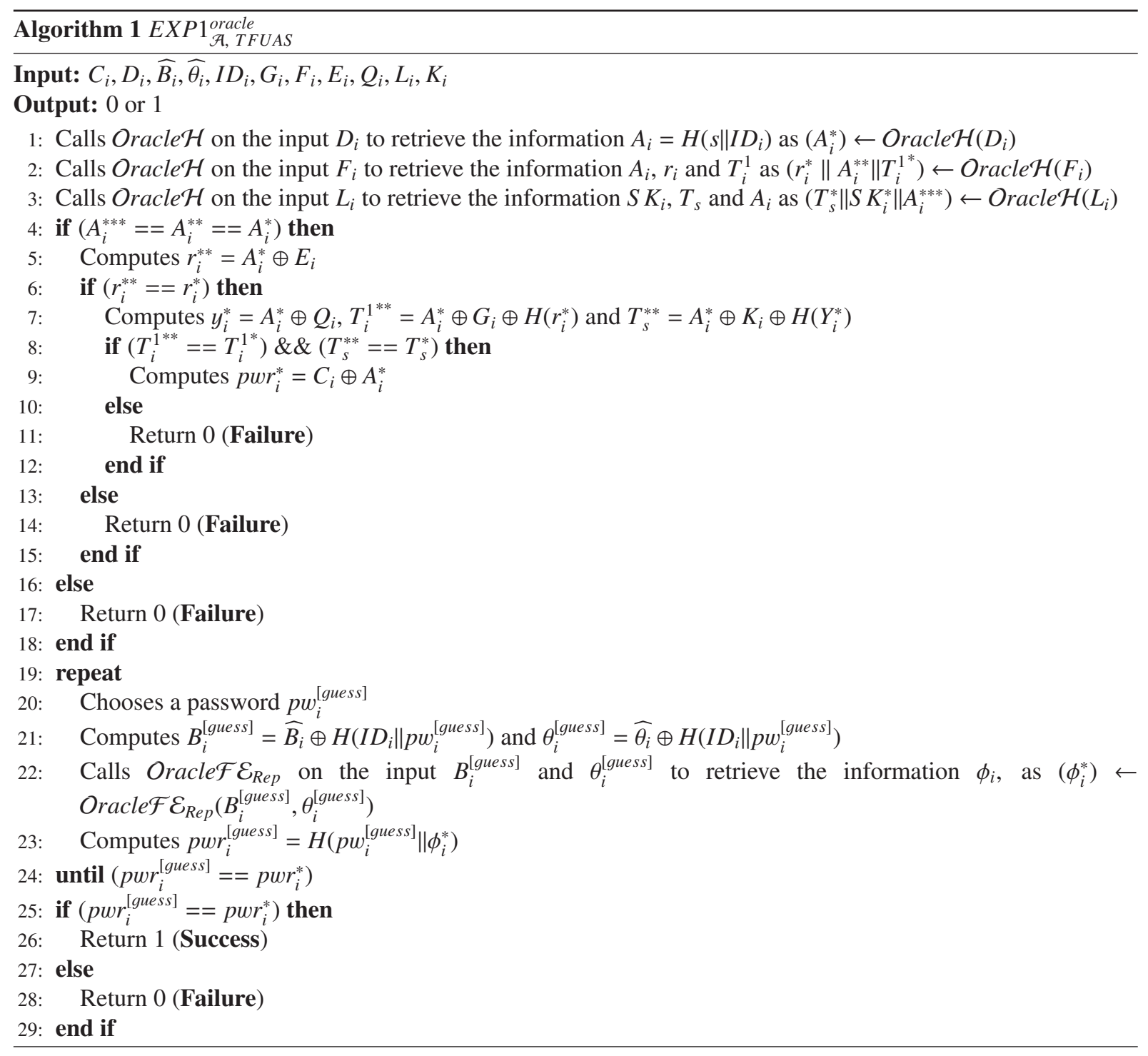

We define the success probability for $E X P 1_{\mathcal{A}, T F U A S}^{\text {oracle }}$ as $S u c c 1_{\mathcal{A}, T F U A S}^{\text {oracle }}=\operatorname{Pr}\left[E X P 1_{\mathcal{A}, T F U A S}^{\text {oracle }}=1\right]$. Then the advantage of $E X P 1_{\mathcal{A}, T F U A S}^{\text {oracle }}$ is given by $A d v 1_{\mathcal{A}, T F U A S}^{\text {oracle }}(t, q H, q F E)=\max _{\mathcal{A}}\left\{S\right.$ ucc $\left.1_{\mathcal{A}, T F U A S}^{\text {oracle }}\right\}$, where the maximum is taken over all $\mathcal{A}$ with the execution time $t$, the number of queries $q H$ made to the Oracle $\mathcal{H}$ oracle and the number of queries $q F E$ made to the $\operatorname{Oracle} \mathcal{F} \mathcal{E}$. Our proposed scheme is said to be provably secure against the adversary $\mathcal{A}$ for deriving the password $p w_{i}$ and biometric parameter $B_{i}$ of a user $U_{i}$, if $A d v 1_{\mathcal{A}, T F U A S}^{\text {oracle }}(t, q H, q F E)$ $\leq \xi$, for any small $\xi>0$. According to algorithm $E X P 1_{\mathcal{A}, T F U A S}^{\text {oracle }}$ (see Algorithm 1 ), if the adversary $\mathcal{A}$ gets success to compute inversion of the cryptographic one-way hash function $H(\cdot)$ and also gets success to solve hardness of fuzzy extractor, he/she can successfully derive the password $p w_{i}$ and biometric parameter $B_{i}$ of the user $U_{i}$ by using of the $O$ racle $\mathcal{H}$ random oracle and $\operatorname{Oracle} \mathcal{F} \mathcal{E}$ random oracle, and wins the game. But, according to Definition 1 and Definition 2, we know that $A d v_{\mathcal{A}}^{\text {Oracle } \mathcal{H}}(t) \leq \xi_{1}$, for any small $\xi_{1}>0$ and $A d v_{\mathcal{A}}^{\text {Oracle } \mathcal{F} \mathcal{E}}(t) \leq \xi_{2}$, for any small $\xi_{2}>0$. Since, we get $A d v 1_{\mathcal{A}, T F U A S}^{\text {oracle }}(t, q H, q F E) \leq \xi$, for any small $\xi>0$ because, the proposed scheme depends on both $A d v_{\mathcal{A}}^{\text {Oracle } \mathcal{H}}(t)$ and $A d v_{\mathcal{A}}^{\text {Oracle } \mathcal{F}}(t)$. Thus, our proposed scheme is secure against the adversary $\mathcal{A}$ for deriving the password $p w_{i}$ and biometric parameter $B_{i}$ of the user $U_{i}$. 
Theorem 2. Under the assumption that a cryptographic one-way hash function $H(\cdot)$ acts as a random oracle, the proposed scheme is provably secure against an adversary $\mathcal{A}$ for deriving the secret key $s$ of the remote server $R S$ even if the adversary $\mathcal{A}$ gets parameters that are stored into the memory of $U_{i}$ 's smart card and traps the communication messages between a user $U_{i}$ and the remote server $R S$.

Proof 2. This research construct an adversary $\mathcal{A}$ who has the ability to derive the secret key $s$ of the remote server $R S$. For this purpose, this research considers same assumptions as discussed in Theorem 1. The adversary $\mathcal{A}$ runs the experiment, EXP $2_{\mathcal{A}, T F U A S}^{\text {oracle }}$ for our three factor user authentication scheme (TFUAS) to derive the secret key $s$ of the remote server $R S$ as given in the Algorithm 2.

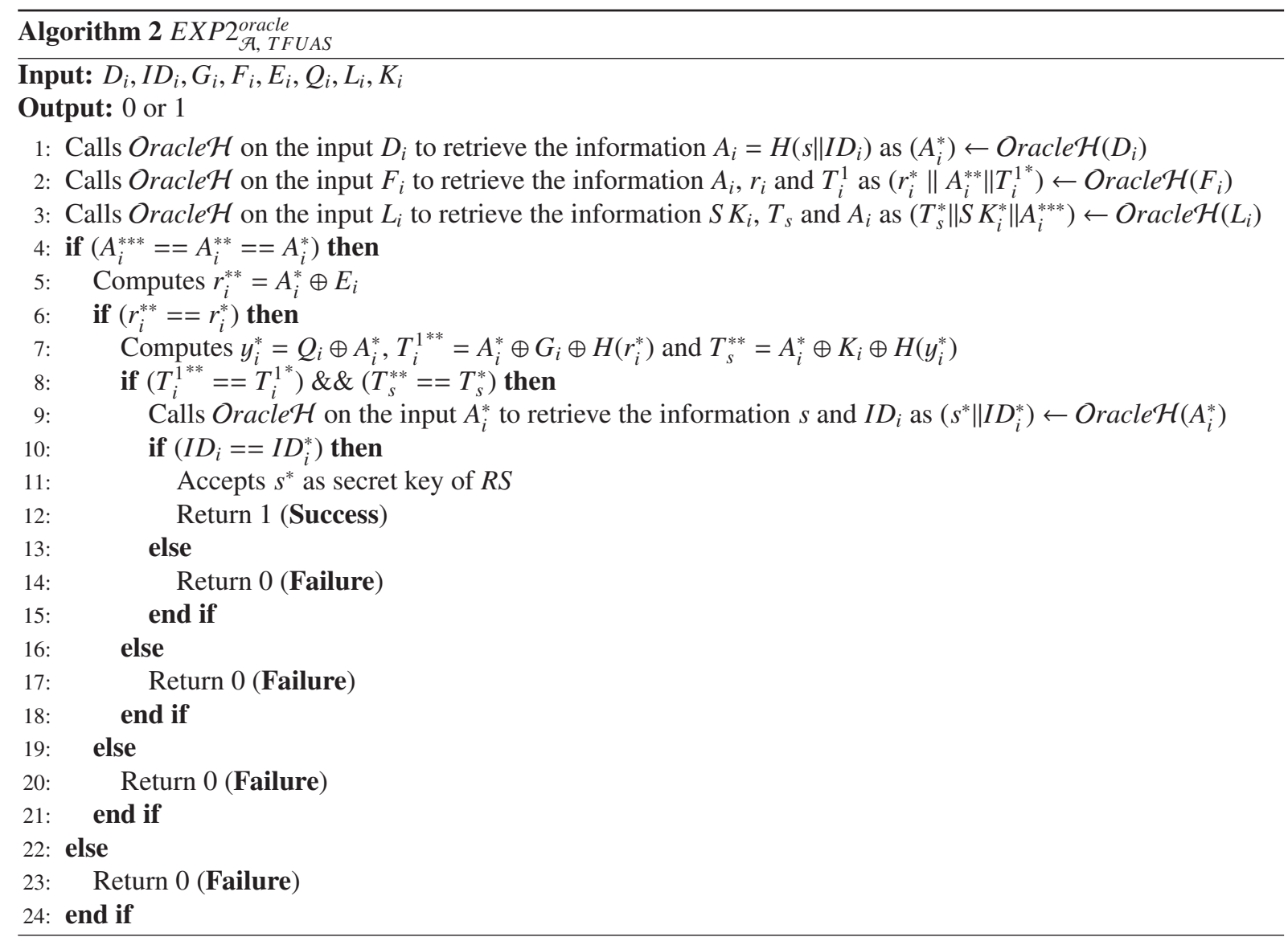

We define the success probability for $E X P 2_{\mathcal{A}, T F U A S}^{\text {oracle }}$ as $S u c c 2_{\mathcal{A}, T F U A S}^{\text {oracle }}=\operatorname{Pr}\left[E X P 2_{\mathcal{A}, T F U A S}^{\text {oracle }}=1\right]$. Then the advantage of $E X P 2_{\mathcal{A}, T F U A S}^{\text {oracle }}$ is given by $A d v 2_{\mathcal{A}, T F U A S}^{\text {oracle }}(t, q H)=\max _{\mathcal{A}}\left\{S u c c 2_{\mathcal{A}, T F U A S}^{\text {oracle }}\right\}$, where the maximum is taken over all $\mathcal{A}$ with the execution time $t$, the number of queries $q H$ made to the Oracle $\mathcal{H}$ oracle. The proposed scheme is said to be provably secure against the adversary $\mathcal{A}$ for deriving the secret key $s$ of the remote server $R S$, if $A d v 2_{\mathcal{A}, T F U A S}^{\text {oracle }}(t, q H) \leq \xi$, for any small $\xi>0$. According to algorithm EXP2 $2_{\mathcal{A}, T F U A S}^{\text {oracle }}$ (see Algorithm 2), if the adversary $\mathcal{A}$ gets success to compute inversion of the cryptographic one-way hash function $H(\cdot)$, he/she can successfully derive the secret key $s$ of the remote server $R S$ by using of the Oracle $\mathcal{H}$ random oracle and wins the game. But, according to Definition 1, we know that $A d v_{\mathcal{H}}^{\text {Oracle } \mathcal{H}}(t) \leq \xi_{1}$, for any small $\xi_{1}>0$. Since, we get $A d v 2_{\mathcal{A}, T F U A S}^{\text {oracle }}(t, q H) \leq \xi$, for any small $\xi>0$ because, the proposed scheme depends on $A d v_{\mathcal{A}}^{\text {Oracle } \mathcal{H}}(t)$. Thus, our proposed scheme is secure against the adversary $\mathcal{A}$ for deriving the secret key $s$ of the remote server $R S$.

Theorem 3. Under the assumption that a cryptographic one-way hash function $H(\cdot)$ acts as a random oracle, the proposed scheme is provably secure against an adversary $\mathcal{A}$ for deriving a shared secret session key $S K_{i}$ between a user $U_{i}$ and the remote server $R S$ even if the adversary $\mathcal{A}$ gets parameters that are stored into the memory of $U_{i}$ 's smart card and traps the communication messages between $U_{i}$ and the remote server $R S$.

Proof 3. This research construct an adversary $\mathcal{A}$ who has the ability to derive the session key $S K_{i}$ between a user $U_{i}$ and the remote server $R S$. For this purpose, this research considers same assumptions as discussed 
in Theorem 1. The adversary $\mathcal{A}$ runs the experiment, EXP $3_{\mathcal{A}, T F U A S}^{\text {oracle }}$ for our three factor user authentication scheme (TFUAS) to derive the session key $S K_{i}$ between the user $U_{i}$ and the remote server $R S$ as given in the Algorithm 3.

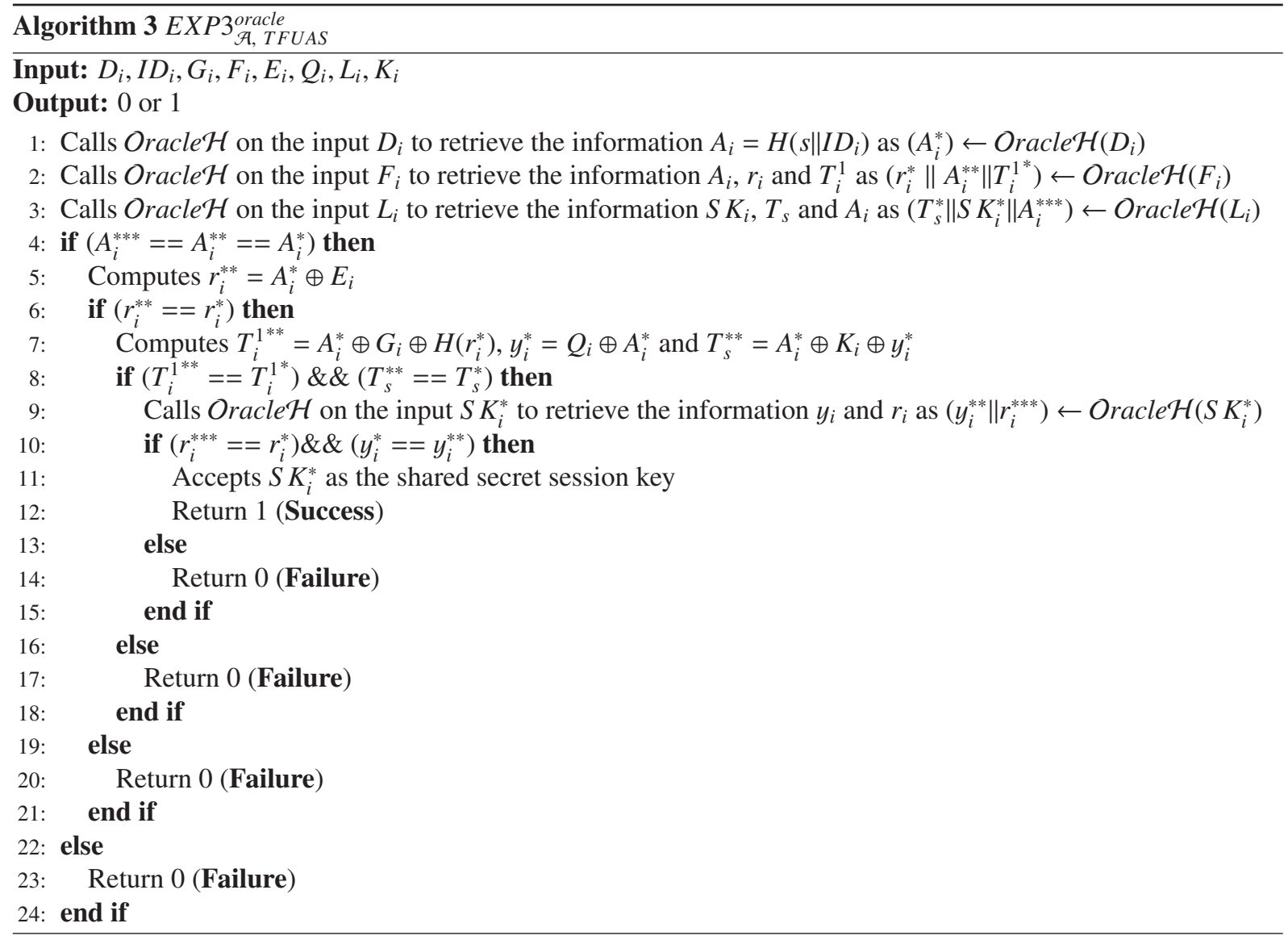

We define the success probability for $E X P 3_{\mathcal{A}, T F U A S}^{\text {oracle }}$ as $S$ ucc $3_{\mathcal{A}, T F U A S}^{\text {oracle }}=\operatorname{Pr}\left[E X P 3_{\mathcal{A}, T F U A S}^{\text {oracle }}=1\right]$. Then the advantage of $E X P 3_{\mathcal{A}, T F U A S}^{\text {oracle }}$ is given by $A d v 3_{\mathcal{A}, T F U A S}^{\text {oracle }}(t, q H)=\max _{\mathcal{A}}\left\{S u c c 3_{\mathcal{A}, T F U A S}^{\text {oracle }}\right\}$, where the maximum is taken over all $\mathcal{A}$ with the execution time $t$, the number of queries $q H$ made to the Oracle $\mathcal{H}$ oracle. The proposed scheme is said to be provably secure against the adversary $\mathcal{A}$ for deriving the session key $S K_{i}$ between the user $U_{i}$ and the remote server $R S$, if $A d v 3_{\mathcal{A}, T F U A S}^{\text {oracle }}(t, q H) \leq \xi$, for any small $\xi>0$. According to algorithm EXP3 $3_{\mathcal{A}, T F U A S}^{\text {oracle }}$ (see Algorithm 3), if the adversary $\mathcal{A}$ gets success to compute inversion of the cryptographic one-way hash function $H(\cdot)$, he/she can successfully derive the session key $S K_{i}$ between the user $U_{i}$ and the remote server $R S$ by using of the Oracle $\mathcal{H}$ random oracle and wins the game. But, according to Definition 1, we know that $A d v_{\mathcal{A}}^{\text {Oracle } \mathcal{H}}(t) \leq \xi_{1}$, for any small $\xi_{1}>0$. Since, we get $A d v 3_{\mathcal{A}, T F U A S}^{\text {oracle }}(t, q H) \leq \xi$, for any small $\xi>0$ because, the proposed scheme depends on $A d v_{\mathcal{A}}^{\text {Oracle } \mathcal{H}}(t)$. Thus, our proposed scheme is secure against the adversary $\mathcal{A}$ for deriving the session key $S K_{i}$ between the user $U_{i}$ and the remote server $R S$.

Theorem 1 demonstrated that the proposed scheme is secure against the off-line password guessing attack. Theorem 3 demonstrates that the proposed scheme is secure against the session key recovery attack because, without knowing random numbers $\left\{r_{i}, y_{i}\right\}$ then $\mathcal{A}$ cannot compute the session key $S K_{i}$. In the proposed scheme, the communicating messages depend on random numbers and the timestamp. Therefore, the communication messages are guaranteed to be different for every session. Thus, $\mathcal{A}$ cannot mount a replay attack on this proposed scheme. In this proposed scheme, $\mathcal{A}$ cannot mount a forgery attack without knowing secret password $p w_{i}$ and biometric parameter $B_{i}$ of a user $U_{i}$ and the secret key $s$ of the remote server $R S$. Theorems 1 and 2 show that the secret information of the remote server and the user are secure from $\mathcal{A}$. Thus, it is infeasible to mount a forgery attack on this proposed scheme.

A valid user say, $U_{\widehat{A}}$ as an adversary $\widehat{A}$ cannot login into the proposed authentication scheme as an another user say, $U_{i}$ because, to login into the system, $\widehat{A}$ has to know the secret key $s$ of the remote server $R S$. As, $\widehat{A}$ is a valid user, it knows their identity $I D_{\widehat{A}}$, password $p w_{\widehat{A}}$ and biometric information $\phi_{\widehat{A}}$. Therefore, $\widehat{A}$ can compute 
Table 2. Security Functionality comparison of the proposed scheme with related schemes

\begin{tabular}{ccccccccc}
\hline Schemes & Ref. [17] & Ref. [18] & Ref. [19] & Ref. [22] & Ref. [23] & Ref. [24] & Ref. [26] & Our scheme \\
\hline A1 & $\sqrt{ }$ & $\sqrt{ }$ & $\times$ & - & $\sqrt{ }$ & $\sqrt{ }$ & $\times$ & $\times$ \\
A2 & $\sqrt{ }$ & $\sqrt{ }$ & $\times$ & $\times$ & $\times$ & $\sqrt{ }$ & $\times$ & $\times$ \\
A3 & $\sqrt{ }$ & $\sqrt{ }$ & $\sqrt{ }$ & - & - & $\times$ & $\times$ & $\times$ \\
A4 & $\sqrt{ }$ & $\sqrt{ }$ & $\times$ & - & $\sqrt{ }$ & $\times$ & $\times$ & $\times$ \\
A5 & $\sqrt{ }$ & $\sqrt{ }$ & $\sqrt{ }$ & - & - & $\sqrt{ }$ & $\sqrt{ }$ & $\times$ \\
A6 & - & - & $\times$ & $\sqrt{ }$ & - & $\times$ & $\times$ & $\times$ \\
EPC & $\times$ & $\sqrt{ }$ & $\times$ & $\times$ & $\times$ & $\sqrt{ }$ & $\sqrt{ }$ & $\sqrt{ }$ \\
ELP & $\times$ & $\sqrt{ }$ & $\times$ & $\times$ & $\times$ & $\sqrt{ }$ & $\sqrt{ }$ & $\sqrt{ }$ \\
\hline
\end{tabular}

A1: Password guessing attack, A2: Insider attack, A3: Forgery attack, A4: Smart card stolen attack, A5: Replay attack, A6: Denial of Service (DoS) attack, ELP: Efficient login phase, EPC: Efficient password change phase, $\times:$ no, $\sqrt{ }$ : yes

Table 3. Computation, communication and storage cost comparison of the proposed scheme with related schemes

\begin{tabular}{|c|c|c|c|c|}
\hline \multirow{2}{*}{ Schemes } & \multirow[t]{2}{*}{$\begin{array}{l}\text { Storage cost } \\
\text { (in bits) }\end{array}$} & \multirow{2}{*}{$\begin{array}{l}\text { Communication Cost } \\
\quad \text { (in bits) } \\
\text { Login + Authentication }\end{array}$} & \multicolumn{2}{|c|}{ Computation cost } \\
\hline & & & Login & Authentication \\
\hline Ref. [17] & 448 & 576 & $2 T_{H}$ & $5 T_{H}$ \\
\hline Ref. [18] & 576 & 832 & $3 T_{H}$ & $8 T_{H}$ \\
\hline Ref. [19] & 576 & 704 & $3 T_{H}$ & $6 T_{H}$ \\
\hline Ref. [22] & 384 & 576 & $4 T_{H}+1 T_{e n c}$ & $7 T_{H}+1 T_{d e c}$ \\
\hline Ref. [23] & 640 & 960 & $3 T_{H}$ & $8 T_{H}$ \\
\hline Ref. [24] & 800 & 1120 & $4 T_{H}$ & $10 T_{H}+1 T_{e n c}+1 T_{d e c}$ \\
\hline Ref. [26] & 384 & 1216 & $4 T_{H}$ & $7 T_{H}+1 T_{e n c}+1 T_{d e c}$ \\
\hline Proposed scheme & 512 & 832 & $4 T_{H}$ & $6 T_{H}$ \\
\hline
\end{tabular}

$H\left(s \| I D_{\widehat{A}}\right)$ by computing $C_{\widehat{A}} \oplus H\left(p w_{\widehat{A}} \| \phi_{\widehat{A}}\right)$, where $C_{\widehat{A}}$ is the smart card store parameter of $\widehat{A}$. From $H\left(s \| I D_{\widehat{A}}\right), \widehat{A}$ cannot extract $s$ due to hardness of inversion of one-way hash function. Furthermore, Theorem 2 shows that $s$ cannot be extracted or computed from known parameters of $\widehat{A}$. Therefore, $\widehat{A}$ unable to mount any attacks on the proposed scheme.

\section{Comparison}

In this section, the performances of the proposed scheme with the existing authentication schemes namely, Li and Hwang's scheme [17], Das's scheme [18], An's scheme [19], Tan's scheme [22], Yan et al.'s scheme [23], Mishra et al.'s schemw [24] and He et al.'s scheme [26] are compared. However, the compared schemes in [17-19] and [22-24] and [26] are not suitable for practical use because, the schemes cannot resist the possible attacks as shown in Table 2. In the introduction part of this paper, it has been described that is insecure against security attacks. Moreover, security analysis of the proposed scheme (see Section 5) shows that the proposed scheme can resist all the possible attacks. Thus, the proposed scheme is more secure than other schemes.

Table 3 shows the computational cost, storage cost and communication overhead comparison of schemes in [17-19], [22-24], and [26] with our proposed scheme. For this purpose, only login and authentications phases have been consider due to maximum use. $T_{H}, T_{e n c}$ and $T_{d e c}$ are the time required for hash operation, symmetric key encryption and decryption respectively. The proposed scheme takes time for 10 hashing operations in two phases which is the lower among related and compared schemes in [18, 22-24, 26]. It can be reasonably assumed that the length of $I D_{i}$ and $p w_{i}$ are 64 bits each. Large prime number $q$, cryptographic one-way hash function $H(\cdot)$ like SHA- ${ }^{1}$, symmetric key encryption/decryption like AES with key size 128 bits $^{2}$, random numbers, symmetric key encryption/decryption and timestamp returns 128 bits each. The communication cost of proposed scheme for login message is $(128+128+128+64)=448$ bits and message generated in authentication phase is $(128+128+128)$

\footnotetext{
${ }^{1}$ http://csrc.nist.gov/publications/fips/fips180-4/fips-180-4.pdf

${ }^{2}$ http://nvlpubs.nist.gov/nistpubs/FIPS/NIST.FIPS.197.pdf
} 
$=384$ bits. Therefore, total communication cost is $(448+384)=832$ bits which is lower than existing and compared schemes in $[23,24,26]$. The storage cost of our proposed scheme is $(128+128+128+128)=512$ bits which is also lower than related schemes in [18, 19, 23, 24].

After resisting all possible attacks as shown in Section 5, the proposed scheme provides better trade-off among storage, computational and communication costs than other related existing schemes. Hence, it can be claimed that the proposed scheme is more efficient and secure than other related existing schemes and also it is applicable for practical applications.

\section{- Advantages of proposed scheme}

In the following, the advantages of the proposed scheme have been discussed.

Efficient login phase: If a user $U_{i}$ enters faulty password and faulty identity by some means in login phase of the proposed scheme, the smart easily can detect the wrong inputs before going to generate a login request message. For this purpose, smart card computes $B_{i}^{\prime}=\widehat{B}_{i} \oplus H\left(I D_{i} \| p w_{i}\right)$ and checks $\operatorname{des}\left(B_{i}^{*}, B_{I}^{\prime}\right) \leq \delta d$. If it does not hold, the smart card rejects $U_{i}$; otherwise, it computes $\theta_{i}^{\prime}=\widehat{\theta_{i}} \oplus H\left(I D_{i} \| p w_{i}\right), \phi_{i}^{\prime} \leftarrow \operatorname{Rep}\left(B_{i}^{*}, \theta_{i}^{\prime}\right), p w r_{i}^{\prime}=H\left(p w_{i} \| \phi_{i}^{\prime}\right), A_{i}^{\prime}$ $=C_{i} \oplus p w r_{i}^{\prime}, D_{i}^{\prime}=H\left(A_{i}^{\prime}\right)$ and checks computed $D_{i}^{\prime}$ and stored $D_{i}$ are equal or not. If equality does not hold, the smart card rejects $U_{i}$; otherwise, accepts the password $p w_{i}$ and identity $I D_{i}$ as correct inputs. Therefore, extra communication overhead due to wrong inputs can be avoided in the proposed scheme.

Efficient password change phase: If a user $U_{i}$ enters faulty password and faulty identity by some means in password phase of the proposed scheme, the smart easily can detect the wrong inputs before going to give permission to the user to submit their new password. For this purpose, smart card computes executes the same steps as mentioned above to check the submitted inputs. If provided inputs are correct, then only the smart card give permission to enter new password to $U_{i}$. Furthermore, to change password of a user, smart card does not need to communicate with the remote server. Therefore, communication overhead is also reduced in proposed scheme with efficient wrong input detection.

Mutual authentication: In the proposed scheme, the remote server $R S$ computes and accepts a secret session key $S K_{i}$ after verifying legitimacy of a user $U_{i}$ through login request message and then, $R S$ sends a reply message to the user $U_{i}$. The user $U_{i}$ agrees upon the same secret session key $S K_{i}$ with $R S$ after verifying legitimacy of $R S$ through reply message. Therefore, both way authentication has been done in the proposed scheme. Furthermore, the proposed scheme can resist all the possible attacks (see, Section 5). Hence, the proposed scheme achieves mutual authentication.

\section{Conclusion}

This paper have proposed an authentication scheme. After analyzing the proposed scheme it can be stated that the proposed scheme can overcome the all possible attacks and has better trade-off among computational, storage and communication costs. Therefore, the proposed scheme is suitable for real world online applications.

\section{References}

[1] T. Maitra, Cryptanalysis of A Secure Remote User Authentication Scheme Using Smart Cards, CoRR abs/1502.04820 (2015)

[2] Y. Dodis, L. Reyzin, A. Smith, in Advances in Cryptology - EUROCRYPT 2004 (Springer Berlin Heidelberg, 2004), Vol. 3027 of Lecture Notes in Computer Science, pp. 523-540

[3] L. Lamport, Password Authentication with Insecure Communication, Commun. ACM 24, 770 (1981)

[4] A. Shimizu, T. Horioka, H. Inagaki, A Password Authentication Method for Contents Communications on the Internet, IEICE Tran. Communications E81-B, 1666 (1998)

[5] J. Xu, W.T. Zhu, D.G. Feng, An improved smart card based password authentication scheme with provable security, Computer Standards \& Interfaces 31, 723 (2009)

[6] X. Li, J. Niu, M.K. Khan, J. Liao, An enhanced smart card based remote user password authentication scheme, Journal of Network and Computer Applications 36, 1365 (2013)

[7] T. Maitra, R. Amin, D. Giri, P.D. Srivastava, An Efficient and Robust User Authentication Scheme for Hierarchical Wireless Sensor Networks without Tamper-Proof Smart Card, I. J. Network Security 18, 553 (2016)

[8] O. Mir, T. van der Weide, C.C. Lee, A Secure User Anonymity and Authentication Scheme Using AVISPA for Telecare Medical Information Systems, Journal of Medical Systems 39, 89 (2015) 
[9] D. Giri, T. Maitra, R. Amin, P. Srivastava, An Efficient and Robust RSA-Based Remote User Authentication for Telecare Medical Information Systems, Journal of Medical Systems 39, 145 (2014)

[10] K.H. Yeh, A lightweight authentication scheme with user untraceability, Frontiers of Information Technology \& Electronic Engineering 16, 259 (2015)

[11] C. Guo, C.C. Chang, Chaotic maps-based password-authenticated key agreement using smart cards, Communications in Nonlinear Science and Numerical Simulation 18, 1433 (2013)

[12] R. Amin, T. Maitra, S.P. Rana, An Improvement of Wang. et. al.'s Remote User Authentication Scheme against Smart Card Security Breach, International Journal of Computer Applications 75, 37 (2013)

[13] C.C. Chang, C.Y. Sun, A Secure and Efficient Authentication Scheme for E-coupon Systems, Wireless Personal Communications 77, 2981 (2014)

[14] R. Amin, T. Maitra, D. Giri, An Improved Efficient Remote User Authentication Scheme in Multi-server Environment using Smart Card, International Journal of Computer Applications 69, 1 (2013)

[15] T. Maitra, M.S. Obaidat, S.H. Islam, D. Giri, R. Amin, Security analysis and design of an efficient ECC-based two-factor password authentication scheme, Security and Communication Networks 9, 4166 (2016)

[16] T. Maitra, M.S. Obaidat, R. Amin, S.H. Islam, S.A. Chaudhry, D. Giri, A robust ElGamal-based passwordauthentication protocol using smart card for client-server communication, International Journal of Communication Systems 30 (2017)

[17] C.T. Li, M.S. Hwang, An efficient biometrics-based remote user authentication scheme using smart cards, Journal of Network and Computer Applications 33, 1 (2010)

[18] A. Das, Analysis and improvement on an efficient biometric-based remote user authentication scheme using smart cards, Information Security, IET 5, 145 (2011)

[19] Y. An, Security Analysis and Enhancements of an Effective Biometric-Based Remote User Authentication Scheme Using Smart Cards, Journal of Biomedicine and Biotechnology 2012, Article ID 519723, 1 (2012)

[20] X. Li, J. Niu, M.K. Khan, J. Liao, X. Zhao, Robust three-factor remote user authentication scheme with key agreement for multimedia systems, Security and Communication Networks (2014)

[21] C.C. Lee, C.W. Hsu, A secure biometric-based remote user authentication with key agreement scheme using extended chaotic maps, Nonlinear Dynamics 71, 201 (2013)

[22] Z. Tan, An efficient biometrics-based authentication scheme for telecare medicine information systems, Przeglad Elektrotechniczny pp. 200-204 (2013)

[23] X. Yan, W. Li, P. Li, J. Wang, X. Hao, P. Gong, A Secure Biometrics-based Authentication Scheme for Telecare Medicine Information Systems, Journal of Medical Systems 37, 9972 (2013)

[24] D. Mishra, S. Mukhopadhyay, A. Chaturvedi, S. Kumari, M. Khan, Cryptanalysis and Improvement of Yan et al.'s Biometric-Based Authentication Scheme for Telecare Medicine Information Systems, Journal of Medical Systems 38, 24 (2014)

[25] T. Maitra, D. Giri, An Efficient Biometric and Password-Based Remote User Authentication using Smart Card for Telecare Medical Information Systems in Multi-Server Environment, Journal of Medical Systems 38, 142 (2014)

[26] D. He, N. Kumar, J.H. Lee, R. Sherratt, Enhanced three-factor security protocol for consumer USB mass storage devices, Consumer Electronics, IEEE Transactions on 60, 30 (2014)

[27] D. Giri, R.S. Sherratt, T. Maitra, R. Amin, Efficient biometric and password based mutual authentication for consumer USB mass storage devices, IEEE Transactions on Consumer Electronics 61, 491 (2015)

[28] D. Giri, R.S. Sherratt, T. Maitra, A novel and efficient session spanning biometric and password based threefactor authentication protocol for consumer USB Mass Storage Devices, IEEE Transactions on Consumer Electronics 62, 283 (2016)

[29] H.F. Huang, H.W. Chang, P.K. Yu, Enhancement of Timestamp-based User Authentication Scheme with Smart Card, International Journal of Network Security 16, 463 (2014)

[30] R. Amin, T. Maitra, D. Giri, P.D. Srivastava, Cryptanalysis and Improvement of an RSA Based Remote User Authentication Scheme Using Smart Card, Wireless Personal Communications (2017)

[31] D. Dolev, A.C. Yao, On the security of public key protocols, Information Theory, IEEE Transactions on 29 , 198 (1983)

[32] P. Kocher, J. Jaffe, B. Jun, Differential Power Analysis, in Advances in Cryptology “CRYPTO'99 (1999), Vol. 1666 of Lecture Notes in Computer Science, pp. 388-397

[33] T.S. Messerges, E.A. Dabbish, R.H. Sloan, Examining Smart-Card Security Under the Threat of Power Analysis Attacks, IEEE Trans. Comput. 51, 541 (2002) 
[34] S.K. Sood, A.K. Sarje, K. Singh, A secure dynamic identity based authentication protocol for multi-server architecture, Journal of Network and Computer Applications 34, 609 (2011) 